\title{
Screening for autism in preterm children: diagnostic utility of the Social Communication Questionnaire
}

\author{
Samantha Johnson, ${ }^{1}$ Chris Hollis, ${ }^{2}$ Enid Hennessy, ${ }^{3}$ Puja Kochhar, ${ }^{2}$ Dieter Wolke, ${ }^{4}$ \\ Neil Marlow ${ }^{1}$
}

${ }^{1}$ Research Department of Academic Neonatology, Institute for Women's Health, University College London, London, UK

${ }^{2}$ Division of Psychiatry, School of Community Health Sciences, University of Nottingham, Nottingham, UK ${ }^{3}$ Wolfson Institute of Preventive Medicine, Barts and The London School of Medicine and Dentistry, Queen Mary, University of London, London, UK

${ }^{4}$ Department of Psychology and Health Sciences Research Institute, Warwick Medical

School, University of Warwick Coventry, UK

\section{Correspondence to} Dr Samantha Johnson, Institute for Women's Health, UCL, 86-96 Chenies Mews, London WC1E 6HX, UK; sam.johnson@nottingham. ac.uk

Accepted 30 August 2010 Published Online First 27 October 2010

\begin{abstract}
Objective Preterm survivors are at high risk for autism spectrum disorders (ASD). The diagnostic utility of the Social Communication Questionnaire (SCO) in screening for ASD was assessed in extremely preterm children at 11 years of age.
\end{abstract}

Design All babies born at $<26$ weeks gestation in UK and Ireland from March through December 1995 were recruited to the EPICure Study. Of 307 survivors, 219 (71\%) were assessed at 11 years. Parents of 173 children completed the SCO to screen for autistic features and the Development and Well Being Assessment (DAWBA) psychiatric interview. A consensus diagnosis of ASD was assigned by two child psychiatrists following review of the DAWBA parental interview and corresponding DAWBA teacher questionnaire.

Setting Community-based follow-up.

Results Using the established SCO cut-off (scores $\geq 15), 28$ (16\%) extremely preterm children screened positive for ASD. Eleven (6\%) were assigned a diagnosis of ASD. Using this cut-off, the SCO had $82 \%$ sensitivity and $88 \%$ specificity for identifying ASD in this population. Using a receiver operating characteristic curve, SCO scores $\geq 14$ had optimal diagnostic utility (area under curve: 0.94; sensitivity: $91 \%$; specificity: $86 \%)$. Positive predictive value was relatively low $(31 \%)$ resulting in numerous over-referrals. However, children with false positive screens had significantly worse neuro-developmental, cognitive and behavioural outcomes than those with true negative screens.

Conclusion The SCO has good diagnostic utility for identifying ASD in extremely preterm children and is a useful screening tool in this population. Children with false positive screens represent a high-risk group in whom further diagnostic assessment would be beneficial.

Extremely preterm birth is associated with a high risk of functional disability later in life. ${ }^{1}$ While neuro-sensory impairments contribute to the range of residual disabilities observed, the most common adverse outcomes are cognitive impairment, behavioural problems and executive dysfunction. ${ }^{2-4}$ Awareness has also increased of a high prevalence of social and communication difficulties and autism spectrum disorders (ASD) in this population. ${ }^{5}{ }^{6}$ Contemporary studies of very preterm survivors have reported positive screening for autistic features in $21-25 \%$ in infancy. ${ }^{7}$ 8 Most recently, we have reported an increased prevalence of autism spectrum symptoms and $8 \%$ prevalence of ASD diagnoses in extremely preterm children at 11 years of age. ${ }^{9}$

\section{What is already known on this topic}

- Extremely preterm children are at high risk for autism spectrum disorders (ASD) in middle childhood.

- The specificity of screening for autism may be confounded by the high prevalence of neurodevelopmental disability in this population.

\section{What this study adds}

- The Social Communication Questionnaire has good diagnostic utility and is a useful first line screening tool for ASD in extremely preterm children.

- Children with false positive screens are at high risk for other neuro-cognitive and behavioural impairments and further neuropsychological assessment would be beneficial.

The importance of screening for ASD in extremely preterm children is increasingly recognised for both clinical and research purposes. Although early intervention improves outcome, screening in infancy is confounded by the high prevalence of neuro-developmental delay in this population. ${ }^{710}$ Screening in middle childhood has greater discriminative validity and many extremely preterm children may present with emerging social and behavioural difficulties prompting the need for screening and assessment at this age. The prevalence of autistic features is also increasingly included as an outcome measure in many epidemiological studies for which cost and time efficient measures are required.

The Social Communication Questionnaire (SCQ $)^{11}$ has been validated for use in middle childhood and has good discriminative validity for identifying children with ASD in clinical samples, ${ }^{12} 13$ those with special educational needs ${ }^{14-16}$ and the normal population. ${ }^{14}$ However, the efficacy of the SCQ in identifying children at risk for ASD has not been investigated in an extremely preterm population in which the high rate of neuro-developmental disability may diminish the predictive validity of such measures. Our aim was therefore to investigate the diagnostic utility of the SCQ in identifying ASD in extremely preterm children in middle childhood. 


\section{METHODS}

\section{Participants}

All babies born at $\leq 25$ weeks gestational age in the UK and Ireland from March through December 1995 were recruited to the EPICure Study, a prospective whole-population study of outcome following extremely preterm birth. Of 307 survivors at 11 years of age, $11(4 \%)$ lived outside the study area, the parents of $57(19 \%)$ declined consent and $18(6 \%)$ did not respond to study invitations to participate. The remaining 219 children ( $71 \%$ of survivors) were formally assessed at 11 years of age (median 131 months; range 121-145 months). Drop-out analyses revealed that children not assessed $(n=89)$ at 11 years were more likely to be born at 25 weeks to unemployed parents of non-white ethnic origin and have more frequent cognitive impairment at 2.5 and 6 years than those assessed $(n=219)$. Detailed drop-out analyses have been published previously. ${ }^{3}$

\section{Measures}

The SCQ (lifetime form) $)^{11}$ is a 40 -item parent report based on the Autism Diagnostic Interview-Revised. Item scores are summed to yield a total SCQ score (range 0-39 for verbal and 0-33 for non-verbal children) with higher scores indicating a greater frequency of symptoms. Missing values were prorated (if $\leq 3$ items were missing on the Social Interaction and Communication subscales and $\leq 2$ items on the Repetitive Behaviour subscale; $\mathrm{n}=16, \mathrm{n}=23, \mathrm{n}=8$, respectively). $\mathrm{SCQ}$ scores were compared to established cut-offs for screening for ASD (scores $\geq 15$ ) and for more narrowly defined autistic disorder (scores $\geq 22$ ). ${ }^{11} 12$

To identify children with ASD diagnoses, parents completed the Development and Well Being Assessment (DAWBA), ${ }^{17}$ a diagnostic interview for childhood psychiatric disorders. Parents were interviewed over the telephone $(88 \%)$ or completed the DAWBA online (12\%). Supplemental information was provided by teachers who completed a corresponding questionnaire-based version of the DAWBA, an approach previously evaluated in a large community study. ${ }^{18}$ Data were entered into an electronic database and scoring algorithms were used to yield computer-generated diagnoses. These computer-generated summary sheets and detailed transcripts of parental descriptions of the child's past and current levels of social and communicative behaviours and play/activities/ routines were reviewed by two child and adolescent psychiatrists who assigned clinical consensus diagnoses based on all

Table 1 Characteristics of extremely preterm children with complete Social Communication Questionnaire (SCQ) and Development and Well-Being Assessment (DAWBA) data and those with incomplete data at 11 years of age

\begin{tabular}{lccl}
\hline Characteristics & $\begin{array}{l}\text { Complete data } \\
(\mathbf{n = 1 7 3 )}\end{array}$ & $\begin{array}{l}\text { Incomplete data } \\
(\mathbf{n}=\mathbf{4 6})\end{array}$ & $\mathbf{p}$ Value \\
\hline Male, $\mathrm{n}(\%)$ & $78(45 \%)$ & $23(50 \%)$ & 0.62 \\
Gestational age $\leq 24$ weeks, $\mathrm{n}(\%)$ & $71(41 \%)$ & $22(48 \%)$ & 0.50 \\
Neuromotor impairment, $\mathrm{n}(\%)$ & $12(7 \%)$ & $9(20 \%)$ & 0.020 \\
Hearing impairment, $\mathrm{n}(\%)$ & $3(2 \%)$ & $1(2 \%)$ & 1.000 \\
Visual impairment, $\mathrm{n}(\%)$ & $11(6 \%)$ & $8(17 \%)$ & 0.034 \\
MPC (IQ) score*, mean (SD) & $85.7(16.5)^{*}$ & $76.1(21.2)$ & 0.006 \\
Cognitive impairment, $\mathrm{n}(\%)$ & $63(36 \%)$ & $24(52 \%)$ & 0.063 \\
Functional disability, $\mathrm{n}(\%)$ & $71(41 \%)$ & $27(59 \%)$ & 0.045 \\
\hline
\end{tabular}

${ }^{*} \mathrm{MPC}$ refers to Mental Processing Composite scores from the Kaufman Assessment Battery for Children. MPC scores were obtained for 171 children with complete data. Definitions of neuromotor, hearing and visual impairment and functional disability have been published elsewhere. ${ }^{1}$ available information. The following DSM-IV-TR ${ }^{19}$ diagnoses in the broad category of ASD were assigned: autistic disorder, Asperger disorder, Rett syndrome, childhood disintegrative disorder and pervasive developmental disorder-not otherwise specified.

Children were also assessed using the Kaufman-Assessment Battery for Children, ${ }^{20}$ a standardised IQ test that yields a Mental Processing Composite (MPC) score (mean 100; SD 15). Cognitive impairment (scores $<-2$ SD) was classified using the mean (SD) of a comparison group of 153 classmates also assessed as part of the EPICure Study ${ }^{3}$ in order to account for the secular increase in IQ scores over time. ${ }^{21}$ Functional neuromotor, hearing and visual impairment was also classified using a standard paediatric evaluation, and overall functional disability was classified using the child's rating in each of the four domains (cognition, hearing, vision, motor). Definitions of functional disability and related outcomes are detailed elsewhere. ${ }^{1}$

Parents completed the Strengths and Difficulties Questionnaire (SDQ)22 to screen for other behavioural and emotional disorders. SDQ scores $>90$ th percentile of the comparison group were used to identify clinically significant emotional symptoms, conduct problems, attention/hyperactivity, peer problems and total difficulties.

Parents and children received study information sheets and informed consent was provided by parents. Children were assessed by a paediatrician and psychologist. Parents completed questionnaires approximately 1 week prior to the child's assessment. The DAWBA was completed subsequently. Psychologists simultaneously scored standardised tests from which excellent inter-rater reliability was achieved: $>95 \%$ agreement in test items. The study was approved by the Southampton and South West Hampshire Research Ethics Committee.

\section{Statistical analyses}

Data were double-entered and analysed using SPSS and Stata. Differences between children with and without complete SCO and DAWBA data were analysed using independent samples $t$ tests for continuous outcomes and Fisher's exact tests for categorical outcomes. To determine diagnostic utility of the SCO, rates of ASD diagnoses and positive SCQ screens were crosstabulated and agreement assessed using Cohen's $\kappa$. Sensitivity, specificity, positive predictive value (PPV) and negative predictive value (NPV) with $95 \%$ CIs were calculated and a receiver operating characteristic (ROC) curve was constructed to identify an SCQ score with optimal diagnostic utility.

\section{RESULTS}

Both SCQ and DAWBA data were obtained for 173 (79\%) children assessed at 11 years (mean age 130 months; range 121-144 months). Of the 46 children with no or incomplete data (nonresponders), the parents of 28 completed the DAWBA only, 10 the SCQ only, and eight did not complete either measure. Nonresponders were significantly more likely to have serious neuromotor (OR 3.26; 95\% CI 1.28 to 8.32) or visual (OR 3.10; 1.17 to 8.24) impairment, functional disability including cognitive impairment (OR 2.04; 1.06 to 3.95) and lower IQ scores (mean difference -9.6 points; $95 \%$ CI -16.4 to -2.9 ; table 1 ).

\section{SCO screens}

The mean total SCQ score was 7.99 (SD 7.51; range 0-35; $\mathrm{n}=173)$. Using the established cut-off score $(\geq 15), 28(16.2 \%)$ 
children screened positive for ASD (figure 1). Boys were significantly more likely than girls to screen positive for ASD (23.1\% vs $10.5 \%$; OR 2.55 ; $95 \%$ CI 1.10 to 5.91 ), and children with cognitive (28.6\% vs 9.1\%; OR 4.00 ; $95 \%$ CI 1.71 to 9.35 ), neuromotor (41.7\% vs $14.3 \%$; OR $4.29 ; 95 \%$ CI 1.25 to 14.66 ) and visual impairment (48.5\% vs $14.2 \%$; OR 5.04; 95\% CI 1.42 to 17.87 ) were significantly more likely to screen positive than those without functional impairment.

\section{ASD diagnoses}

Overall, 11 (6.4\%) extremely preterm children received an ASD diagnosis. Of these, nine were diagnosed with autistic disorder and two with atypical autism. No children were diagnosed with Asperger syndrome or other ASD. Prevalence and correlates of ASD diagnoses for the whole population are published elsewhere. 923

\section{Diagnostic utility}

Mean SCQ scores were significantly higher for children with ASD diagnoses ( $n=11$; mean 23.27; SD 8.32) than those without

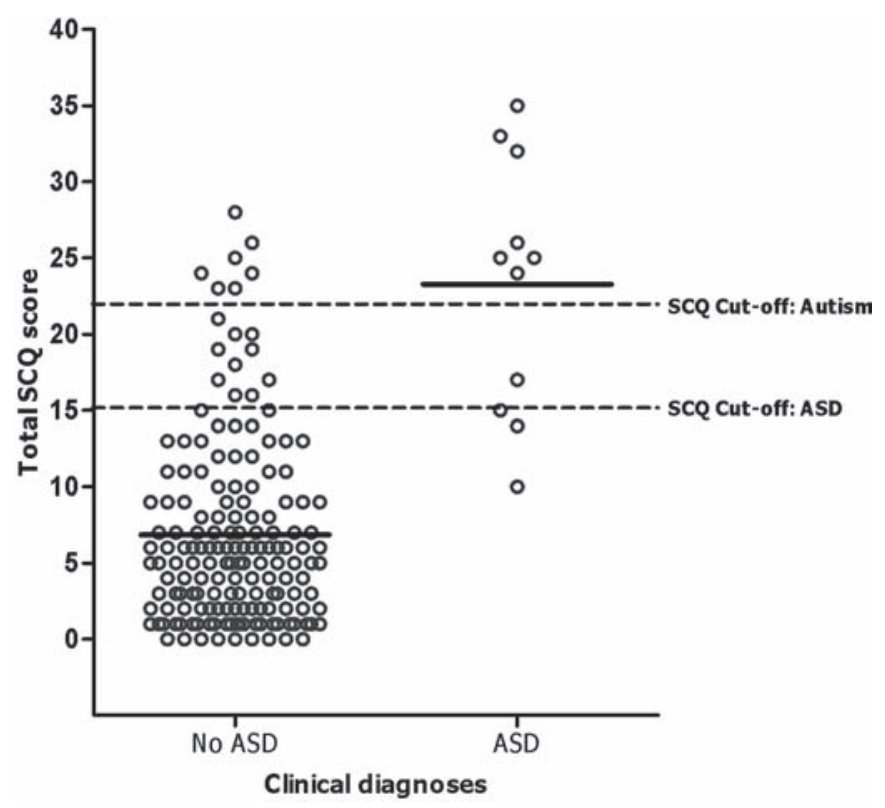

Figure 1 Total Social Communication Questionnaire (SCQ) scores for extremely preterm children with $(n=11)$ and without $(n=162)$ an autism spectrum disorder (ASD) diagnosis at 11 years of age. Clinical diagnoses were assigned using the Development and Well Being Assessment (DAWBA) diagnostic interview. Horizontal bars indicate the mean SCO score. Dashed lines indicate published SCO cut-offs for positive screening.
( $\mathrm{n}=162$; mean 6.85 ; SD 6.22; mean difference 16.43 points; $95 \%$ CI 12.51 to 20.34; figure 1). Agreement between rates of positive SCQ screens and ASD diagnoses were cross-tabulated and predictive values calculated using various SCQ cut-off scores. Using the established SCQ cut-off $(\geq 15)$, there was a significant association between positive SCQ screens and ASD diagnoses ( $\kappa=0.41 ; 95 \%$ CI 0.21 to $0.50 ; \mathrm{p}<0.001)$. While sensitivity $(0.82 ; 95 \%$ CI 0.48 to 0.98$)$ and specificity $(0.88 ; 95 \%$ CI 0.82 to 0.93$)$ was high, PPV was relatively low $(0.32 ; 95 \%$ CI 0.16 to 0.52 ) indicating a large number of false positives: $11 \%$ of all children (table 2).

We examined the predictive value of using a cut-off score of $\geq 22$, usually applied for discriminating between autistic disorder and other ASD, as the default cut-off in this population. Using this cut-off, there was significant agreement between positive SCQ screens and ASD diagnoses $(\kappa=0.53 ; 95 \% \mathrm{CI}$ 0.25 to $0.74 ; \mathrm{p}<0.001)$. However, while PPV was marginally improved $(0.50 ; 95 \%$ CI 0.23 to 0.77$)$ this was at the expense of test sensitivity $(0.64 ; 95 \%$ CI 0.31 to 0.89 ; table 2$)$. The number of false positive classifications was reduced by almost twothirds, but four of the 11 children with ASD diagnoses (57\%) now had false negative SCQ screens and would therefore not be identified as at-risk on screening alone.

An ROC curve was constructed to identify an SCQ cut-off score with maximal predictive value in this population. The area under the curve (AUC) was 0.94 and a cut-off score $\geq 14$ had optimum diagnostic utility $(\kappa=0.41$; sensitivity $0.91,95 \%$ CI 0.59 to 0.99 ; specificity $0.86,95 \%$ CI 0.80 to 0.91$)$. PPV remained relatively low (PPV $0.31 ; 95 \%$ CI 0.16 to 0.50 ), but sensitivity was maximised and all but one child with an ASD diagnosis screened positive on the SCQ.

In high-risk populations, children with false positive scores may be at increased risk for other neuro-developmental impairments, behavioural problems and socio-communication difficulties. Therefore, neuro-developmental outcomes were compared between children with false positive and true negative screens using the ROC-determined cut-off of $\geq 14$ (table 3 ). Children with false positive screens had significantly lower MPC scores than children with true negative screens $(-19.46$ points; $95 \%$ CI -28.32 to -10.59 ; $\mathrm{p}<0.001)$ and were significantly more likely to have functional disabilities (OR 4.11; $95 \%$ CI 1.57 to 10.76 ) including neuromotor, visual and cognitive impairments (table 3 ). Children with false positive screens were also significantly more likely to have parent-reported behaviour problems overall (OR 8.00; 95\% CI 2.90 to 22.04) and in each of the four domains of emotional, conduct, attention and peer problems (table 3).

Excluding children with serious functional disabilities, those with false positive screens had significantly lower MPC scores

Table 2 Effect of SCQ cut-off points for identifying ASD in extremely preterm children $(n=173)$ at 11 years of age

\begin{tabular}{|c|c|c|c|c|c|c|}
\hline \multirow[b]{2}{*}{$\begin{array}{l}\text { SC } 0 \text { cut-off } \\
\text { score }\end{array}$} & \multicolumn{2}{|c|}{$\begin{array}{l}\text { Number of positive } \\
\text { screens }\end{array}$} & \multicolumn{4}{|l|}{ Predictive values } \\
\hline & $\begin{array}{l}\text { ASD } \\
(n=11)\end{array}$ & $\begin{array}{l}\text { No ASD } \\
(n=162)\end{array}$ & Sensitivity (95\% Cl) & Specificity (95\% CI) & PPV (95\% Cl) & NPV (95\% Cl) \\
\hline$\geq 14^{*}$ & 10 & 22 & $91 \%(59 \%$ to $99 \%)$ & $86 \%(80 \%$ to $91 \%)$ & $31 \%(16 \%$ to $50 \%)$ & $99 \%(96 \%$ to $100 \%)$ \\
\hline$\geq 15^{\dagger}$ & 9 & 19 & $82 \%(48 \%$ to $98 \%)$ & $88 \%(82 \%$ to $93 \%)$ & $32 \%(16 \%$ to $52 \%)$ & $99 \%(95 \%$ to $100 \%)$ \\
\hline$\geq 22$ & 7 & 7 & $64 \%(31 \%$ to $89 \%)$ & $96 \%$ (91\% to $98 \%$ ) & $50 \%(23 \%$ to $77 \%)$ & $99 \%(94 \%$ to $99 \%)$ \\
\hline
\end{tabular}

ASD, autism spectrum disorder; NPV, negative predictive value; PPV, positive predictive value; SC0, Social Communication Questionnaire.

* Optimal cut-off in this population.

${ }^{\dagger}$ Optimal established cut-off. 
Table 3 Differences in neuro-developmental outcomes between extremely preterm children with true negative and false positive SCO screens using a total SCO cut-off score of $\geq 14$

\begin{tabular}{|c|c|c|c|c|}
\hline \multirow[b]{2}{*}{ Outcome } & \multicolumn{2}{|l|}{ SC0 screen n (\%) } & \multirow[b]{2}{*}{ OR $(95 \%$ CI) } & \multirow[b]{2}{*}{ p Value } \\
\hline & True negative $(n=140)$ & False positive $(n=22)$ & & \\
\hline \multicolumn{5}{|l|}{ Functional impairment } \\
\hline Neuromotor impairment, $\mathrm{n}(\%)$ & $7(5.0 \%)$ & $4(18.2 \%)$ & $4.22(1.12$ to 15.86$)$ & 0.045 \\
\hline Visual impairment, $\mathrm{n}(\%)$ & $6(4.3 \%)$ & $4(18.2 \%)$ & $4.96(1.28$ to 19.29$)$ & 0.031 \\
\hline Hearing impairment, $\mathrm{n}(\%)$ & $1(0.7 \%)$ & $1(4.5 \%)$ & $6.62(0.40$ to 109.89$)$ & 0.254 \\
\hline Cognitive impairment, n (\%) & $41(29.3 \%)$ & $15(68.2 \%)$ & $5.17(2.00$ to 113.62$)$ & 0.001 \\
\hline MPC (IO) scores, mean (SD) & $89.14(14.26)$ & $69.68(19.36)$ & $-19.46(-28.32 \text { to }-10.59)^{*}$ & 0.000 \\
\hline Overall functional disability, $\mathrm{n}(\%)$ & $48(34.3 \%)$ & $15(68.2 \%)$ & 4.11 (1.57 to 10.76$)$ & 0.004 \\
\hline \multicolumn{5}{|l|}{ Parent SDQ: clinical range ${ }^{\dagger}$} \\
\hline Total difficulties, n (\%) & $35(25.0 \%)$ & $16(72.7 \%)$ & $8.00(2.90$ to 22.04$)$ & 0.000 \\
\hline Emotional symptoms, n (\%) & $21(15.0 \%)$ & $11(50.0 \%)$ & $5.67(2.18$ to 14.74$)$ & 0.001 \\
\hline Conduct problems, $\mathrm{n}(\%)$ & $12(8.6 \%)$ & $6(27.3 \%)$ & $4.0(1.32$ to 12.13$)$ & 0.020 \\
\hline Hyperactivity/inattention, n (\%) & $28(20.0 \%)$ & $16(72.75)$ & 10.67 (3.83 to 29.75$)$ & 0.000 \\
\hline Peer problems, n (\%) & $30(21.4 \%)$ & $13(59.1 \%)$ & 5.30 (2.07 to 13.57$)$ & 0.001 \\
\hline \multicolumn{5}{|c|}{$\begin{array}{l}\text { *Mean difference }(95 \% \mathrm{CI}) \text {. } \\
{ }^{+} \text {Behavioural outcomes were assessed using the Strengths and Difficulties Questionnaire (SDO) completed by parents } \\
\text { ( } \mathrm{n}=162 \text { ). Children with scores > } 90 \text { th percentile of a comparison group of } 153 \text { classmates were classified as at risk for } \\
\text { clinically significant difficulties in each domain and overall for total difficulties. } \\
\text { MPC, Mental Processing Composite scores from the Kaufman Assessment Battery for Children; SCQ, Social Communication }\end{array}$} \\
\hline
\end{tabular}

(mean difference -7.55 points; $95 \% \mathrm{CI}-14.55$ to -0.55 ) and were more likely to have emotional (OR 20.50; 95\% CI 3.51 to $119.92 ; \mathrm{p}=0.001)$, conduct (OR $13.05 ; 95 \%$ CI 2.27 to 74.90 ; $\mathrm{p}=0.011$ ), attention/hyperactivity (OR $11.88 ; 95 \%$ CI 2.11 to $66.73 ; p=0.004$ ) and peer problems (OR 26.47; 95\% CI 2.99 to $234.52 ; p=0.001)$ than those with true negative screens.

\section{DISCUSSION}

Recent reports of a high prevalence of ASD in extremely preterm children ${ }^{9}$ highlight the need for screening for social and communication difficulties in these survivors. This study shows that the SCQ has good diagnostic utility for identifying ASD in extremely preterm children.

The mean SCQ score (mean 8; SD 8) and rate of positive screens in this study were higher than those reported for normal population samples ${ }^{14} 24$ and reflect the generally higher level of autism spectrum symptoms in extremely preterm survivors. ${ }^{9} 25$ Using the established cut-off score for identifying ASD ( $\geq 15)$, the SCQ had high sensitivity (82\%) and specificity (88\%) exceeding standards required for screening tests. ${ }^{26}$ These values are comparable with or exceed estimates reported for diagnostic utility of the SCQ in clinical samples ${ }^{12}$ 13 and in children with special educational needs ${ }^{14-16}$ in middle childhood, and far exceed values reported for diagnostic utility of the SCQ in younger children. ${ }^{27-29}$ Construction of a ROC curve produced a high AUC (0.94) and a total SCQ cutoff score of $\geq 14$ was found to have optimal diagnostic utility maximising both sensitivity (91\%) and specificity (86\%) in this population.

Assessing sensitivity and specificity alone can be misleading. In practice, professionals may have screening test results alone on which to base decisions for referral and thus SCQ predictive values should be considered. In this population, NPV were consistently high ( $\geq 98 \%$ ), thus assuring the clinician or researcher that almost all children with negative screens would fail to meet diagnostic criteria for ASD. However, PPV were relatively low: $31-32 \%$ of children with positive screens received an ASD diagnosis resulting in a high rate of false positive screens $(13 \%$ of all children screened). A high rate of false positives is not uncommon in behavioural screening in which PPVs of $30-50 \%$ are often reported. ${ }^{26} 30 \mathrm{SCO}$ scores are elevated in children with learning disabilities and behavioural problems in the absence of diagnosed ASD, ${ }^{12} 31$ and such disorders are among the most prevalent adverse outcomes associated with extreme prematurity. ${ }^{2} 3$ This study shows that children with functional disabilities are four times more likely to screen positive on the SCQ than their extremely preterm peers. Parents who lack a conceptual understanding of the nature and aetiology of ASD may be unable to discriminate these symptoms and may rate SCQ items as positive based on behaviours associated with other neuro-developmental sequelae. ${ }^{15}$

Over-referrals are considered a negative consequence of screening if the cost of further diagnostic assessment is high and confers no benefit. However, routine screening is likely to be cost effective in this population as those with false positive scores were four to six times more likely to have cognitive or neuro-sensory impairment, and eight times more likely to have parent-reported behaviour problems than those with true negative screens. Even after exclusion of children with serious neuro-sensory disabilities, those with false positive screens were over 20 times more likely to have anxiety or peer problems. Thus those with false positive screens are a group in whom further diagnostic assessment and psychiatric referral would be beneficial.

Our analysis of the characteristics of non-responders showed that parents of children with functional disabilities, particularly physical and neuro-sensory impairments, were less likely to complete the SCQ. Such parents may feel that some items are not applicable or are difficult to answer when trying to isolate autistic features from other neuro-developmental sequelae. Professionals using the SCQ with extremely preterm cohorts should be aware of this response bias.

The strengths of the present study lie in the investigation of the efficacy of the SCQ in a whole-population-based cohort of extremely preterm children including rigorous assessments of neuro-psychological outcomes. Psychiatric interviews were carried out for all children in the study using the DAWBA and consensus diagnoses were made by two experienced child 
psychiatrists using information gained in the DAWBA including parent and teacher descriptions of behaviour. The DAWBA is a well-established diagnostic interview that was used as the principal measure of childhood psychopathology in the British Mental Health Surveys ${ }^{32}$ and as a specific diagnostic measure in population-based prevalence studies of ASD, ${ }^{33} 34$ generating prevalence estimates comparable to other ASD diagnostic measures. The validity of the DAWBA diagnosis of ASD is also supported by evidence of good agreement $(\kappa=0.75)$ between DAWBA diagnosis and the Diagnostic Interview for Social and Communicative Disorders. ${ }^{35}$ Parents completed the SCQ prior to the DAWBA so that that questionnaire responses would be unaffected by participation in the interview. SCQ results were not affected by chronological age as reflected by the lack of a significant correlation between SCQ scores and age at assessment $(r=0.029 ; p=0.705 ; n=173)$. Given the association between age and SCQ scores, ${ }^{13}$ the diagnostic utility of the SCO in this population may differ if used at other ages. This warrants further investigation.

\section{CONCLUSIONS}

The SCQ has good diagnostic utility for identifying extremely preterm children with ASD and is an effective method of firstlevel screening in this population. Children with false positive screens are at high risk for other neuro-behavioural sequelae and thus further diagnostic assessment and educational planning is warranted. The SCQ is a useful screening tool and a cost effective outcome measure for assessing autistic spectrum symptomatology in extremely preterm children.

\begin{abstract}
Acknowledgements The authors are indebted to the EPICure Study Group, which includes paediatricians in $\mathbf{2 7 6}$ maternity units in the UK and Ireland who identified the cohort, contributed perinatal data and whose help was invaluable. We are also indebted to the children and parents for their continued participation in the EPICure Study. Data collection was conducted by Joe Fawke, Sue Thomas, Vicky Rowell, Rebecca Smith, Rebecca Trikic and Samantha Johnson. Support for DAWBA assessments was provided by Robert Goodman (London). Co-investigators for the EPICure Studies were Neil Marlow (UCL; Principal Investigator), Kate Costeloe (Queen Mary, University of London), Enid Hennessy (Queen Mary, University of London), Janet Stocks (UCL) and Elizabeth Draper (University of Leicester). The EPICure study website can be viewed at www.epicure.ac.uk.
\end{abstract}

Funding This study was funded by the Medical Research Council (MRC), UK. Neil Marlow receives a proportion of funding from the Department of Health's NIHR Biomedical Research Centres funding scheme at UCLH/UCL.

\section{Competing interests None.}

Ethics approval This study was conducted with the approval of the Southampton and South West Hampshire Research Ethics Committee.

Provenance and peer review Not commissioned; externally peer reviewed.

\section{REFERENCES}

1. Johnson S, Fawke J, Hennessy E, et al. Neurodevelopmental disability through 11 years of age in children born before 26 weeks of gestation. Pediatrics 2009;124:e249-57.

2. Samara M, Marlow N, Wolke D. Pervasive behavior problems at 6 years of age in a total-population sample of children born at $</=25$ weeks of gestation. Pediatrics 2008;122:562-73.

3. Johnson S, Hennessy E, Smith R, et al. Academic attainment and special educational needs in extremely preterm children at 11 years of age: the EPICure study. Arch Dis Child Fetal Neonatal Ed 2009;94:F283-9.

4. Marlow N, Hennessy EM, Bracewell MA, et al. Motor and executive function at 6 years of age after extremely preterm birth. Pediatrics 2007;120:793-804.

5. Elgen I, Sommerfelt K, Markestad T. Population based, controlled study of behavioural problems and psychiatric disorders in low birthweight children at 11 years of age. Arch Dis Child Fetal Neonatal Ed 2002;87:F128-32.

6. Indredavik MS, Vik T, Heyerdahl S, et al. Psychiatric symptoms and disorders in adolescents with low birth weight. Arch Dis Child Fetal Neonatal Ed 2004;89:F445-50.
7. Kuban KC, O'Shea TM, Allred EN, et al. Positive screening on the Modified Checklist for Autism in Toddlers (M-CHAT) in extremely low gestational age newborns. J Pediatr 2009;154:535-540.e1.

8. Limperopoulos C, Bassan H, Sullivan NR, et al. Positive screening for autism in ex-preterm infants: prevalence and risk factors. Pediatrics 2008;121:758-65.

9. Johnson S, Hollis C, Kochhar P, et al. Autism spectrum disorders in extremely preterm children. J Pediatr 2010;156:525-31.e2.

10. Johnson S, Marlow N. Positive screening results on the modified checklist for autism in toddlers: implications for very preterm populations. J Pediatr 2009;154:478-80.

11. Rutter M, Bailey A, Lord C. The Social Communication Questionnaire. Los Angeles, CA: Western Psychological Services, 2003.

12. Berument SK, Rutter M, Lord C, et al. Autism screening questionnaire: diagnostic validity. Br J Psychiatry 1999;175:444-51.

13. Corsello C, Hus V, Pickles A, et al. Between a ROC and a hard place: decision making and making decisions about using the SCO. J Child Psychol Psychiatry 2007;:48:932-40

14. Chandler S, Charman T, Baird G, et al. Validation of the social communication questionnaire in a population cohort of children with autism spectrum disorders. J Am Acad Child Adolesc Psychiatry 2007;46:1324-32.

15. Charman T, Baird G, Simonoff E, et al. Efficacy of three screening instruments in the identification of autistic-spectrum disorders. Br J Psychiatry 2007;191:554-9

16. Witwer AN, Lecavalier L. Autism screening tools: an evaluation of the Social Communication Questionnaire and the Developmental Behaviour Checklist-Autism Screening Algorithm. J Intellect Dev Disabil 2007;32:179-87.

17. Goodman R, Ford T, Richards H, et al. The Development and Well-Being Assessment: description and initial validation of an integrated assessment of child and adolescent psychopathology. J Child Psychol Psychiatry 2000;41:645-55

18. Ford T, Goodman R, Meltzer H. The British Child and Adolescent Mental Health Survey 1999: The prevalence of DSM-IV disorders. J Am Acad Child Adolesc Psychiatry2003;42:1203-11.

19. American Psychiatric Association. Diagnostic and Statistical Manual of Mental Disorders. Fourth Edition. Text Revision. Washington, DC: American Psychiatric Association 2000.

20. Kaufman AS, Kaufman NL. Kaufman Assessment Battery for Children. Circle Pines, MN: American Guidance Service, Inc, 1983

21. Wolke D, Ratschinski G, Ohrt B, et al. The cognitive outcome of very preterm infants may be poorer than often reported: an empirical investigation of how methodological issues make a big difference. Eur J Pediatr 1994;153:906-15.

22. Goodman R. The Strengths and Difficulties Questionnaire: a research note. J Child Psychol Psychiatry 1997;38:581-6.

23. Johnson S, Hollis C, Kochhar P, et al. Psychiatric disorders in extremely preterm children: Iongitudinal finding at age 11 years in the EPICure study. J Am Acad Child Adolesc Psychiatry 2010;49:453-63.e1.

24. Mulligan A, Richardson T, Anney RJ, et al. The Social Communication Questionnaire in a sample of the general population of school-going children. Ir J Med Sci 2009;178:193-9.

25. Hack M, Taylor HG, Schluchter M, et al. Behavioral outcomes of extremely low birth weight children at age 8 years. J Dev Behav Pediatr 2009;30:122-30.

26. Glascoe FP. Screening for developmental and behavioral problems. Ment Retard Dev Disabil Res Rev 2005;11:173-9.

27. Eaves LC, Wingert $\mathrm{H}, \mathrm{Ho}$ HH. Screening for autism: agreement with diagnosis. Autism 2006;10:229-42.

28. Allen CW, Silove N, Williams K, et al. Validity of the social communication questionnaire in assessing risk of autism in preschool children with developmental problems. J Autism Dev Disord 2007;37:1272-8.

29. Lee L, David AB, Rusyniak J, et al. Performance of the Social Communication Questionnaire in children receiving preschool special education services. Res Autism Spectr Disord2007;1:126-38.

30. Johnson S, Wolke D, Marlow N. Developmental assessment of preterm infants at 2 years: validity of parent reports. Dev Med Child Neurol 2008;50:58-62

31. Towbin KE, Pradella A, Gorrindo T, et al. Autism spectrum traits in children with mood and anxiety disorders. J Child Adolesc Psychopharmacol 2005;15:452-64.

32. Green H, McGinnity A, Meltzer H, et al. Mental Health of Children and Young People in Great Britain. Basingstoke, Hampshire: National Statistics, 2005.

33. Fombonne E, Simmons H, Ford T, et al. Prevalence of pervasive developmental disorders in the British nationwide survey of child mental health. J Am Acad Child Adolesc Psychiatry 2001;40:820-7.

34. Heiervang $\mathbf{E}$, Stormark KM, Lundervold AJ, et al. Psychiatric disorders in Norwegian 8- to 10-year-olds: an epidemiological survey of prevalence, risk factors, and service use. J Am Acad Child Adolesc Psychiatry 2007;46:438-47.

35. Posserud M, Lundervold AJ, Lie SA, et al. The prevalence of autism spectrum disorders: impact of diagnostic instrument and non-response bias. Soc Psychiatry Psychiatr Epidemiol 2010;45:319-27. 